\title{
Linear and Nonlinear Stability Analysis of Double Diffusive Convection in a Maxwell Fluid Saturated Porous Layer with Internal Heat Source
}

\author{
Moli Zhao, ${ }^{1}$ Qiangyong Zhang, ${ }^{1}$ and Shaowei Wang ${ }^{2}$ \\ ${ }^{1}$ Geotechnical and Structural Engineering Research Center, Shandong University, Jinan, Shandong 250061, China \\ ${ }^{2}$ Department of Engineering Mechanics, School of Civil Engineering, Shandong University, Jinan, Shandong 250061, China \\ Correspondence should be addressed to Shaowei Wang; shaoweiwang@sdu.edu.cn
}

Received 7 January 2014; Revised 19 March 2014; Accepted 19 March 2014; Published 9 April 2014

Academic Editor: Chongbin Zhao

Copyright ( $) 2014$ Moli Zhao et al. This is an open access article distributed under the Creative Commons Attribution License, which permits unrestricted use, distribution, and reproduction in any medium, provided the original work is properly cited.

\begin{abstract}
The onset of double diffusive convection is investigated in a Maxwell fluid saturated porous layer with internal heat source. The modified Darcy law for the Maxwell fluid is used to model the momentum equation of the system, and the criterion for the onset of the convection is established through the linear and nonlinear stability analyses. The linear analysis is obtained using the normal mode technique, and the nonlinear analysis of the system is studied with the help of truncated representation of Fourier series. The effects of internal Rayleigh number, stress relaxation parameter, normalized porosity, Lewis number, Vadasz number and solute Rayleigh number on the stationary, and oscillatory and weak nonlinear convection of the system are shown numerically and graphically. The effects of various parameters on transient heat and mass transfer are also discussed and presented analytically and graphically.
\end{abstract}

\section{Introduction}

Double diffusive convection in porous media without heat source has been intensively studied because of its application in different branches of science and engineering, such as underground disposal of nuclear wastes, groundwater pollution, contaminant transport in fluid-saturated soils, liquid gas storage, and food processing [1-4]. Since convective flow plays an important role in ore-forming systems, Zhao et al. have conducted extensive and systematic studies on double diffusive convection within the upper crust of the Earth during the last decade or so [5-9]. Their work has promoted the better understanding of ore-forming mechanisms, which are essential to explore new large ore deposits in the deep Earth $[8,9]$. And the onset of convection due to internal heat source has become an interesting problem in various areas of geophysics and engineering under the situations of radioactive decay or a weak exothermic reaction within the porous material. Therefore, diffusive convection in porous media with internal heat source has attracted the attention of many authors like Nield and Bejan [10], Ingham and Pop [11], and Vafai [12] during the last several decades.
The onset of thermodynamic instability in horizontal porous layer saturated with Newtonian fluid was first studied extensively on geological and engineering length scales [13-23]. Convection by internal heat sources has been studied in several papers including experimental and theoretical studies. The onset of convection in a fluid saturated horizontal layer of an anisotropic porous medium with internal heat source subjected to inclined temperature gradient has been considered [24]. The effect of internal heat source on the problem of triple diffusive convection has been analyzed by Straughan and Tracey [25]. Magyari et al. have carried out an analytical and numerical study about the effect of boundary-layer flows in a fluid-saturated porous medium with internal heat generation [26, 27]. Hill has investigated linear and nonlinear stability analyses of double diffusive convection in a fluid saturated porous layer with internal concentration source [28]. Bhadauria et al. have studied the linear and nonlinear thermal instability in an anisotropic saturated porous layer with internal heat source [29]. Bhadauria has made the stability analysis of convection in a binary fluid-saturated horizontal porous layer with internal heat source [30]. 
Recently, viscoelastic fluid flow in porous media has attracted considerable attention, due to the large demands of such diverse fields as biorheology, geophysics, chemical industries, and petroleum industries. Wang and Tan have made the stability analysis of double diffusive convection in a Maxwell fluid saturated porous medium [31]. It is worthwhile to point out that the first viscoelastic rate type model, which is still used widely, is due to Maxwell. The onset of double diffusive convection in a viscoelastic saturated porous layer has been considered by many researchers (e.g., [31-36]). However, we are unaware of the double diffusive problem in any previous work which also introduces penetrative convection with internal heat source.

In this paper, we focus on the linear and weakly nonlinear stability analyses in a viscoelastic fluid saturated porous layer with internal heat source using the Darcy-Maxwell model. The Dufour and Soret effects are ignored. The aim of the present paper is to study how onset criteria for stationary and oscillatory double diffusive convection are affected by the viscoelastic parameter and other parameters, as well as discussing their effects on heat and mass transfer.

\section{Mathematical Model}

Assuming that an infinite horizontal porous layer saturated with Maxwell fluid mixture with internal heat source, confined between the planes, $z=0$ and $z=d$. A Cartesian frame of reference is chosen with the origin in the lower boundary and the $z$-axis vertically upwards. For the purposes of the present paper, we consider that temperature difference $\Delta T$ and concentration difference $\Delta S$ are maintained between the lower and upper boundaries. In the Boussinesq approximation, the equation of state is

$$
\rho=\rho_{0}\left[1-\alpha_{T}\left(T-T_{0}\right)+\alpha_{S}\left(S-S_{0}\right)\right],
$$

where $T$ is the temperature, $S$ is the concentration, $T_{0}$ and $S_{0}$ are temperature and concentration at the above plate, $\alpha_{T}$ and $\alpha_{S}$ are thermal and solutal expansion coefficients in the medium, $\rho$ is the density of fluid, and $\rho_{0}$ is the density at some reference temperature $T_{0}$ and concentration $S_{0}$.

Considering the vertically downward gravity force $\mathbf{g}$ acting on it and neglecting the off-diagonal (Soret, Dufour, and cross-diffusion) contributions to the fluxes of the stratifying agencies, by using of the modified Darcy-Maxwell model [35], the governing system for double diffusive of Maxwell fluid in a porous layer with internal heat source can be represented by

$$
\begin{gathered}
\nabla \cdot \mathbf{q}=0, \\
\left(1+\lambda_{1} \frac{\partial}{\partial t}\right)\left(\frac{\rho_{0}}{\varepsilon} \frac{\partial \mathbf{q}}{\partial t}+\nabla p-\rho \mathbf{g}\right)+\frac{\mu}{K} \mathbf{q}=0, \\
\gamma \frac{\partial T}{\partial t}+\mathbf{q} \cdot \nabla T=\kappa_{T} \nabla^{2} T+Q\left(T-T_{0}\right), \\
\varepsilon \frac{\partial S}{\partial t}+\mathbf{q} \cdot \nabla S=\kappa_{S} \nabla^{2} S .
\end{gathered}
$$

The thermal boundary conditions are

$$
\begin{aligned}
& T=T_{0}+\Delta T \quad \text { at } z=0, \quad T=T_{0} \quad \text { at } z=d, \\
& S=S_{0}+\Delta S \quad \text { at } z=0, \quad S=S_{0} \quad \text { at } z=d,
\end{aligned}
$$

where $\mathbf{q}=(u, v, w)$ is the Darcy velocity, $p$ is the pressure, $\lambda_{1}$ is the relaxation time, $Q$ is internal heat source, $\mathbf{g}$ is the gravitational acceleration, $\mu$ is the viscosity, while $K$ and $\varepsilon$ are the permeability and porosity of the medium, and $\gamma=(\rho c)_{m} /(\rho c)_{f}$ is the ratio of heat capacities, $\kappa_{T}$ and $\kappa_{S}$ are effective thermal and solutal diffusivity of the medium, respectively.

The basic state of the fluid is assumed to be quiescent, and the quantities of the basic state are given by

$$
\mathbf{q}_{b}=\mathbf{0}, \quad p=p_{b}(z), \quad T=T_{b}(z), \quad S=S_{b}(z),
$$

which satisfy the following conditions:

$$
\begin{gathered}
\frac{d p_{b}}{d z}=-\rho_{b} g, \quad \frac{d^{2} S_{b}}{d z^{2}}=0, \\
\kappa_{T} \frac{d^{2}\left(T_{b}-T_{0}\right)}{d z^{2}}+Q\left(T_{b}-T_{0}\right)=0 .
\end{gathered}
$$

Here the subscript $b$ refers to the basic state. Then the steady state solutions are given by

$$
\begin{gathered}
T_{b}=T_{0}+\Delta T \frac{\sin \sqrt{R_{i}}(1-z / d)}{\sin \sqrt{R_{i}}}, \\
S_{b}=S_{0}+\Delta S\left(1-\frac{z}{d}\right), \\
p_{b}=p_{0}-\rho_{0} g z \\
\times\left(1-\frac{\alpha_{T} \Delta T \sin \sqrt{R_{i}}(1-z / d)}{\sin \sqrt{R_{i}}}+\alpha_{S} \Delta S\left(1-\frac{z}{d}\right)\right),
\end{gathered}
$$

where $R_{i}=Q d^{2} / \kappa_{T}$ is internal Rayleigh number. On the basic state, we superimpose perturbations in the following form:

$$
\begin{gathered}
\mathbf{q}=\mathbf{q}_{b}+\mathbf{q}^{\prime}, \quad p=p_{b}+p^{\prime}, \\
T=T_{b}+T^{\prime}, \quad S=S_{b}+S^{\prime}, \\
\rho=\rho_{b}+\rho^{\prime},
\end{gathered}
$$

where primes indicate perturbed quantities. Substituting (7) into (1)-(2) and using the basic state solutions, we obtain the following governing equations:

$$
\begin{gathered}
\nabla \cdot \mathbf{q}^{\prime}=0, \\
\left(1+\lambda_{1} \frac{\partial}{\partial t}\right)\left(\frac{\rho_{0}}{\varepsilon} \frac{\partial \mathbf{q}^{\prime}}{\partial t}+\nabla p^{\prime}+\rho_{0} \mathbf{g}\left(\alpha_{T} T^{\prime}-\alpha_{S} s^{\prime}\right)\right) \\
+\frac{\mu}{K} \mathbf{q}^{\prime}=0,
\end{gathered}
$$




$$
\begin{gathered}
\gamma \frac{\partial T^{\prime}}{\partial t}+\mathbf{q}^{\prime} \cdot \nabla T^{\prime}+w^{\prime} \frac{\partial T_{b}}{\partial z}=\kappa_{T} \nabla^{2} T^{\prime}+Q T^{\prime}, \\
\varepsilon \frac{\partial S^{\prime}}{\partial t}+\mathbf{q}^{\prime} \cdot \nabla S^{\prime}-w^{\prime} \frac{\Delta S^{\prime}}{d}=\kappa_{S} \nabla^{2} S^{\prime} .
\end{gathered}
$$

Eliminating the pressure term from the momentum equation by using curl-curl operator and introducing the following nondimensional transformation (after ignoring the primes for simplicity):

$$
\begin{array}{cc}
x=x^{*} d, & t=\frac{\gamma d^{2}}{\kappa_{T}} t^{*}, \quad q=\frac{\kappa_{T}}{d} q^{*}, \\
p=\frac{\mu \kappa_{T}}{K} p^{*}, & T=(\Delta T) T^{*}, \quad S=(\Delta S) S^{*},
\end{array}
$$

we obtain the nondimensional governing equations (on dropping the asterisks for simplicity):

$$
\begin{gathered}
\left(1+\lambda \frac{\partial}{\partial t}\right)\left(\frac{\eta}{\operatorname{Va}} \frac{\partial}{\partial t} \nabla^{2} w-\mathrm{Ra}_{1}^{2}(T-N S)\right)+\nabla^{2} w=0 \\
\left(\frac{\partial}{\partial t}-\nabla^{2}-R_{i}\right) T+\mathbf{q} \cdot \nabla T+w f(z)=0 \\
\left(\eta \frac{\partial}{\partial t}-\frac{1}{\mathrm{Le}} \nabla^{2}\right) S+\mathbf{q} \cdot \nabla S-w=0
\end{gathered}
$$

where $\lambda=\lambda_{1} \kappa / \gamma d^{2}$ the relaxation number, $\eta=\varepsilon / \gamma$ the normalized porosity, $\mathrm{Da}=K / d^{2}$ the Darcy number, $\operatorname{Pr}=\mu / \rho_{0} \kappa_{T}$ the Prandtl number, Va $=\varepsilon^{2} \operatorname{Pr} / \mathrm{Da}$ the Vadasz number, $\mathrm{Ra}=\rho_{0} g \alpha_{T} K d \Delta T / \mu \kappa_{T}$ the thermal Rayleigh number, $N=\alpha_{S} \Delta S / \alpha_{T} \Delta T$ the buoyancy ratio, Le $=$ $\kappa_{T} / \kappa_{S}$ the Lewis number, and $f(z)=\partial T_{b} / \partial z$, where $T_{b}$ in nondimensionalized form is given by

$$
T_{b}=\frac{\sin \sqrt{R_{i}}(1-z)}{\sin \sqrt{R_{i}}} .
$$

The boundaries of the system considered here are impermeable isothermal and isosolutal. Hence, the boundary conditions for the perturbation variables are given by

$$
w=T=S=0 \quad \text { at } z=0,1 .
$$

\section{Linear Stability Analysis}

In this section, we discuss the linear stability analysis. According to the normal mode analysis $[8,34]$, convective motion is assumed to exhibit horizontal periodicity. Then the perturbed quantities can be assumed to be periodic waves of the form

$$
\left(\begin{array}{c}
W \\
T \\
S
\end{array}\right)=\left(\begin{array}{c}
W(z) \\
\Theta(z) \\
\Phi(z)
\end{array}\right) \exp [i(l x+m y)+\omega t]
$$

where $l$ and $m$ are the wavenumbers in the horizontal plane and $\omega$ is the growth rate. Infinitesimal perturbations of the rest state may either damp or grow depending on the value of the parameter $\omega$. Substituting (16) into (13) yields

$$
\begin{gathered}
(1+\lambda \omega)\left(\frac{\eta \omega}{\mathrm{Va}}\left(D^{2}-a^{2}\right) W+a^{2} \operatorname{Ra}(\Theta-N \Phi)\right) \\
+\left(D^{2}-a^{2}\right) W=0 \\
W f(z)+\left(-D^{2}+a^{2}+\omega-R_{i}\right) \Theta=0 \\
W+\left(\frac{1}{\mathrm{Le}}\left(D^{2}-a^{2}\right)-\eta \omega\right) \Phi=0
\end{gathered}
$$

where $D=d / d z$ and $a^{2}=l^{2}+m^{2}$. Now, the boundary conditions become

$$
W=\Theta=\Phi=0 \quad \text { at } z=0,1 .
$$

To satisfy the boundary conditions (18), we assume the solutions of (17) in the following form:

$$
\left(\begin{array}{l}
W(z) \\
\Theta(z) \\
\Phi(z)
\end{array}\right)=\left(\begin{array}{l}
W_{0} \\
\Theta_{0} \\
\Phi_{0}
\end{array}\right) \sin n \pi z, \quad(n=1,2,3, \ldots)
$$

which is the most unstable mode when $n=1$, that is, the fundamental mode. Substituting the above equation into (17) yields

$$
\begin{gathered}
(1+\lambda \omega)\left(\frac{\eta \omega}{\mathrm{Va}} \delta^{2} W_{0}-a^{2} \operatorname{Ra}\left(\Theta_{0}-N \Phi_{0}\right)\right)+\delta^{2} W_{0}=0 \\
2 F W_{0}+\left(\delta^{2}+\omega-R_{i}\right) \Theta_{0}=0 \\
W_{0}-\left(\frac{\delta^{2}}{\mathrm{Le}}+\eta \omega\right) \Phi_{0}=0
\end{gathered}
$$

where $\delta^{2}=\pi^{2}+a^{2}$ is the total wave number and $F=$ $\int_{0}^{1} f(z) \sin ^{2} \pi z d z$. Rewrite the above equations in the matrix form as

$$
\begin{aligned}
& \left(\begin{array}{ccc}
\delta^{2}\left(1+\Lambda \frac{\eta \omega}{\mathrm{Va}}\right) & -a^{2} \operatorname{Ra} \Lambda & a^{2} \operatorname{Ra} N \Lambda \\
2 F(z) & \left(\delta^{2}+\omega-R_{i}\right) & 0 \\
1 & 1 & -\left(\frac{\delta^{2}}{\mathrm{Le}}+\eta \omega\right)
\end{array}\right)\left(\begin{array}{l}
W_{0} \\
\Theta_{0} \\
\Phi_{0}
\end{array}\right) \\
& =\left(\begin{array}{l}
0 \\
0 \\
0
\end{array}\right) \text {, }
\end{aligned}
$$

where $\Lambda=1+\lambda \omega$. Considering the nontrivial solution of the above matrix equation, we require

$$
\begin{aligned}
\mathrm{Ra}= & \frac{\delta^{2}(1+\Lambda(\eta \omega / \mathrm{Va}))\left(R_{i}-\delta^{2}-\omega\right)}{2 a^{2} \Lambda F} \\
& +\frac{\left(R_{i}-\delta^{2}-\omega\right) \mathrm{Ra}_{S}}{2 F\left(\delta^{2}+\text { Le } \eta \omega\right)},
\end{aligned}
$$


where $\mathrm{Ra}_{S}=$ RaNLe $=\rho_{0} g \alpha_{S} K d \Delta S / \mu \kappa_{S}$ is the solutal Rayleigh number. For neutral solutions, we set $\omega=i \omega_{i}$ in (22) and rearrange the terms to get the Rayleigh number (dropping the subscript $i$ of $\omega_{i}$ ):

$$
\mathrm{Ra}_{T}=\Delta_{1}+i \omega \Delta_{2},
$$

where $\Delta_{1}$ and $\Delta_{2}$ are real part and imaginary part given by

$$
\begin{aligned}
\Delta_{1}= & \frac{\delta^{2}\left(R_{i}-\delta^{2}-\lambda \omega^{2}+(\eta / \mathrm{Va}) \omega^{2}+\lambda^{2} \eta \omega^{4} / \mathrm{Va}\right)}{2 a^{2} F(z)\left(1+\lambda^{2} \omega^{2}\right)} \\
& +\frac{\operatorname{Ra}_{S}\left(R_{i} \delta^{2}-\delta^{4}-\mathrm{Le} \eta \omega^{2}\right)}{2 F(z)\left(\delta^{4}+\mathrm{Le}^{2} \eta^{2} \omega^{2}\right)}, \\
\Delta_{2}= & \left(\delta ^ { 2 } \left(R_{i} \eta-\eta \delta^{2}-\mathrm{Va}\left(\lambda R_{i}-\lambda \delta^{2}+1\right)\right.\right. \\
& \left.\left.+\lambda^{2} \eta R_{i} \omega^{2}-\lambda^{2} \delta^{2} \eta \omega^{2}\right)\right) \\
& \times\left(2 a^{2} F(z)\left(1+\lambda^{2} \omega^{2}\right) \mathrm{Va}\right)^{-1} \\
& +\frac{\operatorname{Ra}_{S}\left(-\operatorname{Le} \eta R_{i}+\mathrm{Le}^{2} \delta^{2}-\delta^{2}\right)}{2 F(z)\left(\delta^{4}+\mathrm{Le}^{2} \eta^{2} \omega^{2}\right)} .
\end{aligned}
$$

Since $\mathrm{Ra}$ is a physical quantity, it must be real. Hence, from (24) it follows that either $\omega=0$ (steady onset) or $\Delta_{2}=0$ $(\omega \neq 0$, oscillatory onset).

3.1. Stationary Convection. For the validity of principle of exchange of stabilities (i.e., steady case), we have $\omega=0$ at the margin of stability. Then, the Rayleigh number at which marginally stable steady mode becomes

$$
\mathrm{Ra}_{T}^{s t}=-\frac{1}{2 F(z)}\left(\frac{\delta^{2}\left(\delta^{2}-R_{i}\right)}{a^{2}}+\frac{\left(\delta^{2}-R_{i}\right) \mathrm{Ra}_{S}}{\delta^{2}}\right) .
$$

In the absence of the internal heat source; that is, $Q=0$, we have $R_{i}=0$ and $F(z)=-1 / 2$. So we obtain

$$
\mathrm{Ra}_{T}^{s t}=\frac{\left(\pi^{2}+a^{2}\right)^{2}}{a^{2}}+\mathrm{Ra}_{S},
$$

which coincides with the known results $[8,10]$. Furthermore, when $a_{S}=0$, the stationary Rayleigh number reduces to the classical result:

$$
\mathrm{Ra}_{T}^{s t}=\frac{\left(\pi^{2}+a^{2}\right)^{2}}{a^{2}} .
$$

In addition, (26) gives the critical value $\mathrm{Ra}_{T, c}^{s t}=4 \pi^{2}$ for $a_{c}^{s t}=$ $\pi$.

3.2. Oscillatory Convection. For oscillatory onset, $\Delta_{2}=0$ and $\omega \neq 0$, and then

$$
\begin{aligned}
\operatorname{Ra}_{T}^{\text {Osc }}= & \frac{\delta^{2}\left(R_{i}-\delta^{2}-\lambda \omega^{2}+(\eta / \mathrm{Va}) \omega^{2}+\lambda^{2} \eta \omega^{4} / \mathrm{Va}\right)}{2 a^{2} F(z)\left(1+\lambda^{2} \omega^{2}\right)} \\
& +\frac{\operatorname{Ra}_{S}\left(R_{i} \delta^{2}-\delta^{4}-\mathrm{Le} \eta \omega^{2}\right)}{2 F(z)\left(\delta^{4}+\mathrm{Le}^{2} \eta^{2} \omega^{2}\right)}
\end{aligned}
$$

where the frequency $\omega$ in $(28)$ is given by

$$
b_{2}\left(\omega^{2}\right)^{2}+b_{1} \omega^{2}+b_{0}=0 .
$$

Here

$$
\begin{gathered}
b_{2}=\left(\lambda^{2} \eta R_{i}-\lambda^{2} \delta^{2} \eta\right) \operatorname{Le}^{2} \eta^{2} \delta^{2}, \\
b_{1}=\left(R_{i} \eta-\eta \delta^{2}-\operatorname{Va}\left(\lambda R_{i}-\lambda \delta^{2}+1\right)\right) \\
\times \operatorname{Le}^{2} \eta^{2} \delta^{2}+\left(\lambda^{2} \eta R_{i}-\lambda^{2} \delta^{2} \eta\right) \delta^{6} \\
+a^{2} \lambda^{2} \operatorname{VaRa}_{S}\left(-\operatorname{Le} \eta R_{i}+\operatorname{Le} \eta \delta^{2}-\delta^{2}\right), \\
b_{0}=\left(R_{i} \eta-\eta \delta^{2}-\operatorname{Va}\left(\lambda R_{i}-\lambda \delta^{2}+1\right)\right) \delta^{6} \\
+a^{2} \operatorname{VaRa}_{S}\left(-\operatorname{Le} \eta R_{i}+\operatorname{Le} \eta \delta^{2}-\delta^{2}\right) .
\end{gathered}
$$

Now, we try to find the positive solutions of (29). If there is none, then no oscillatory convection instability occurs. If there are two positive roots, then the minimum of (28) with $\omega^{2}$ obtained by (29) gives the oscillatory neutral Rayleigh number. If we find only one positive solution, then substituting $\omega^{2}$ into (27) yields the oscillatory Rayleigh number. After that, the effects of different parameters on the onset of oscillatory convection can be discussed from the Rayleigh number.

\section{Nonlinear Analysis}

In this section, we study the nonlinear stability analysis using minimal truncated Fourier series. For simplicity, we confine ourselves to two-dimensional rolls, so that all the physical quantities are independent of $y$. Defining the stream function $\psi$ such that $u=\partial \psi / \partial z, w=-\partial \psi / \partial x$ and substituting them into (9)-(11) and then eliminating the pressure term, nondimensionalizing the governing equations yields

$$
\begin{gathered}
\left(1+\lambda \frac{\partial}{\partial t}\right)\left(\frac{\eta}{\operatorname{Va}} \frac{\partial}{\partial t}\left(\nabla^{2} \psi\right)+\operatorname{Ra} \frac{\partial T}{\partial x}-\frac{\mathrm{Ra}_{S}}{\operatorname{Le}} \frac{\partial S}{\partial x}\right)+\nabla^{2} \psi=0, \\
\left(\frac{\partial}{\partial t}-\nabla^{2}-R_{i}\right) T=\frac{\partial \psi}{\partial x} f(z)+\frac{\partial(\psi, T)}{\partial(x, z)}, \\
\eta \frac{\partial S}{\partial t}-\frac{\partial(\psi, S)}{\partial(x, z)}=\frac{1}{\mathrm{Le}} \nabla^{2} S-\frac{\partial \psi}{\partial x} .
\end{gathered}
$$

We assume that the basic circulation remains undistorted but the temperature and concentration fields are distorted by the addition of a second harmonic with no $x$-dependence, and thus

$$
\begin{gathered}
\psi=A_{1}(t) \sin (a x) \sin (\pi z), \\
T=B_{1}(t) \cos (a x) \sin (\pi z)+B_{2}(t) \sin (2 \pi z), \\
S=C_{1}(t) \cos (a x) \sin (\pi z)+C_{2}(t) \sin (2 \pi z),
\end{gathered}
$$

where $A_{1}, B_{1}, B_{2}, C_{1}$, and $C_{2}$ are time dependent amplitudes and to be determined from the dynamics of the system. 
Substituting (32)-(34) into the coupled nonlinear system of partial differential equation (31) and equating coefficients for expression terms, we obtain the following nonlinear system:

$$
\frac{d X}{d t}=D
$$

where $X=\left(A_{1}, A_{2}, B_{1}, B_{2}, C_{1}, C_{2}\right)^{T}, D=\left(D_{1}, D_{2}, D_{3}, D_{4}\right.$, $\left.D_{5}, D_{6}\right)^{T}$ with

$$
\begin{gathered}
D_{1}=A_{2}, \\
D_{2}=-\frac{\mathrm{Va}}{\lambda \eta \delta^{2}}\left(\frac{\eta \delta^{2}}{\mathrm{Va}} A_{2}+\delta^{2} A_{1}+a \mathrm{Ra} B_{1}-\frac{a \mathrm{Ra}_{S}}{\mathrm{Le}} C_{1}\right. \\
\left.+a \mathrm{Ra} \lambda D_{3}-\frac{a \lambda \mathrm{Ra}_{S}}{\mathrm{Le}} D_{5}\right), \\
D_{3}=2 a F(z) A_{1}-\pi a A_{1} B_{2}+\left(R_{i}-\delta^{2}\right) B_{1}, \\
D_{4}=\frac{\pi a}{2} A_{1} B_{1}+\left(R_{i}-4 \pi^{2}\right) B_{2}, \\
D_{5}=-\frac{\pi a}{\eta} A_{1} C_{2}-\frac{\delta^{2}}{\eta \mathrm{Le}} C_{1}-\frac{a}{\eta} A_{1}, \\
D_{6}=\frac{\pi a}{2 \eta} A_{1} C_{1}-\frac{4 \pi^{2}}{\mathrm{Le} \eta} C_{2} .
\end{gathered}
$$

The above ordinary differential equations are autonomous, which is difficult to obtain the solutions by use of analytical technique, so we solve the above system numerically. We note that the above system is uniformly bounded in time and possesses many properties to the full problem. Equation (34) must be dissipative. Therefore, the divergence of the flow is always negative. So we have

$$
\begin{aligned}
\frac{\partial \dot{A}_{1}}{\partial A_{1}} & +\frac{\partial \dot{A}_{2}}{\partial A_{2}}+\frac{\partial \dot{B}_{1}}{\partial B_{1}}+\frac{\partial \dot{B}_{2}}{\partial B_{2}}+\frac{\partial \dot{C}_{1}}{\partial C_{1}}+\frac{\partial \dot{C}_{2}}{\partial C_{2}} \\
& =-\left(\frac{1}{\lambda}-2 R_{i}+\left(4 \pi^{2}+\delta^{2}\right)\left(1+\frac{1}{\text { Le } \eta}\right)\right) .
\end{aligned}
$$

The system is bounded and dissipative. Consequently, the trajectories are attracted to a set of measure zero in the phase space. In particular, they may be attracted to a fixed point, a limit cycle or, perhaps, a strange attractor. From (37), it can also be concluded that if a set of initial points in phase space occupy a region $V(0)$ at time $t=0$, then after some time $t$ we have

$$
V(t)=V(0) \exp \left\{-\left[\frac{1}{\lambda}-2 R_{i}+\left(4 \pi^{2}+\delta^{2}\right)\left(1+\frac{1}{\mathrm{Le} \eta}\right)\right] t\right\},
$$

which indicates that the volume decreases exponentially with time. Moreover, we can conclude that an increase in the value of relaxation parameter $\lambda$, Lewis number Le, and normalized porosity $\eta$ is to enhance the dissipation. Furthermore, we observe that (37) has an important symmetry, for it is invariant under the transformation:

$$
\left(A_{1}, A_{2}, B_{1}, B_{2}, C_{1}, C_{2}\right) \longrightarrow\left(-A_{1},-A_{2},-B_{1}, B_{2},-C_{1}, C_{2}\right) \text {. }
$$

4.1. Steady Finite Amplitude Motions. The simplified model represented by (37) has the great advantage that steady finite amplitude solutions can be obtained at once and their stability can be investigated analytically. From qualitative prediction, we look into the possibility of an analytical solution. In the case of steady motions, setting the left hand side of (37) equal to zero yields

$$
\begin{gathered}
\delta^{2} A_{1}+a \mathrm{Ra} B_{1}-\frac{a \mathrm{Ra} s}{\mathrm{Le}} C_{1}=0, \\
2 a F(z) A_{1}-\pi a A_{1} B_{2}+\left(R_{i}-\delta^{2}\right) B_{1}=0, \\
\frac{\pi a}{2} A_{1} B_{1}+\left(R_{i}-4 \pi^{2}\right) B_{2}=0, \\
-\frac{\pi a}{\eta} A_{1} C_{2}-\frac{\delta^{2}}{\eta \mathrm{Le}} C_{1}-\frac{a}{\eta} A_{1}=0, \\
\frac{\pi a}{2 \eta} A_{1} C_{1}-\frac{4 \pi^{2}}{\mathrm{Le} \eta} C_{2}=0 .
\end{gathered}
$$

On solving for the amplitudes in terms of $A_{1}$, we obtain

$$
\begin{gathered}
B_{1}=\frac{2 a F(z)\left(8 \pi^{2}-2 R_{i}\right) A_{1}}{\pi^{2} a^{2} A_{1}^{2}-\left(R_{i}-\delta^{2}\right)\left(8 \pi^{2}-2 R_{i}\right)}, \\
C_{1}=-\frac{8 a \operatorname{Le} A_{1}}{8 \delta^{2}+a^{2} \operatorname{Le}^{2} A_{1}^{2}}, \\
B_{2}=\frac{2 \pi a^{2} A_{1}^{2} F(z)}{\pi^{2} a^{2} A_{1}^{2}-\left(R_{i}-\delta^{2}\right)\left(8 \pi^{2}-2 R_{i}\right)}, \\
C_{2}=-\frac{a^{2} \operatorname{Le}^{2} A_{1}^{2}}{\pi\left(8 \delta^{2}+a^{2} \operatorname{Le}^{2} A_{1}^{2}\right)} .
\end{gathered}
$$

Substituting $B_{1}$ and $C_{1}$ into (39) yields

$$
a_{2} x^{2}+a_{1} x+a_{0}=0,
$$

where $x=A_{1}^{2} / 8$, and

$$
\begin{aligned}
& a_{2}=\delta^{2} M_{2} M_{5}, \\
& a_{1}=\delta^{4} M_{2}-\delta^{2} M_{3} M_{5}+M_{1} M_{5}+M_{2} M_{4}, \\
& a_{0}=M_{1} \delta^{2}-M_{3} M_{4}-M_{3} \delta^{4}, \\
& M_{1}=2 a^{2} \operatorname{Ra} F(z)\left(\pi^{2}-\frac{R_{i}}{4}\right), \\
& M_{2}=\pi^{2} a^{2}, \\
& M_{3}=\left(R_{i}-\delta^{2}\right)\left(\pi^{2}-\frac{R_{i}}{4}\right), \\
& M_{4}=a^{2} \mathrm{Ra}_{S}, \\
& M_{5}=a^{2} \mathrm{Le}^{2} .
\end{aligned}
$$

The required root of (42) is

$$
\frac{A_{1}^{2}}{8}=\frac{-a_{1}+\sqrt{a_{1}^{2}-4 a_{2} a_{0}}}{2 a_{2}} .
$$


If the discriminant equals zero, we obtain the expression for finite amplitude Rayleigh number $\mathrm{Ra}_{T}^{F}$, which characterizes the onset of finite amplitude steady motions. The finite amplitude Rayleigh number can be expressed in the following form:

$$
\mathrm{Ra}_{T}^{F}=\frac{-e_{1}+\sqrt{e_{1}^{2}-4 e_{2} e_{0}}}{2 e_{2}}
$$

where

$$
\begin{aligned}
e_{2}= & a^{8} \operatorname{Le}^{4} F^{2}(z)\left(\pi^{2}-\frac{R_{i}}{4}\right)^{2} \\
& -4 \delta^{4} a^{6} F^{2}(z) \operatorname{Le}^{2}\left(\pi^{2}-\frac{R_{i}}{4}\right)^{2} \\
e_{1}= & 2 a^{4} \operatorname{Le}^{2} F(z)\left(\delta^{4} \pi^{2} a^{2}-\delta^{2} a^{2} \operatorname{Le}^{2}\left(R_{i}-\delta^{2}\right)\right. \\
& \left.\times\left(\pi^{2}-\frac{R_{i}}{4}\right)+\pi^{2} a^{4} \mathrm{Ra}_{S}\right)\left(\pi^{2}-\frac{R_{i}}{4}\right) \\
& +4 \delta^{2} a^{4} F(z) \mathrm{Le}^{2}\left(\pi^{2}-\frac{R_{i}}{4}\right) \\
& \times a^{2} \operatorname{Ra}_{S}\left(R_{i}-\delta^{2}\right)\left(\pi^{2}-\frac{R_{i}}{4}\right) \\
& +\delta^{4}\left(R_{i}-\delta^{2}\right)\left(\pi^{2}-\frac{R_{i}}{4}\right) \\
e_{0}= & \left(\delta^{4} \pi^{2} a^{2}-\delta^{2} a^{2} \mathrm{Le}^{2}\left(R_{i}-\delta^{2}\right)\right. \\
& \left.\times\left(\pi^{2}-\frac{R_{i}}{4}\right)+\pi^{2} a^{4} \mathrm{Ra}_{S}\right)^{2} .
\end{aligned}
$$

4.2. Heat Transfer and Mass Transport. In the study of convection problems, the determination of heat transfer and mass transport play a very important role. Let $H$ and $J$ denote the rate of heat and mass transport per unit for the fluid phase. For

$$
\begin{gathered}
H=-\left.\kappa_{T}\left\langle\frac{\partial T_{\text {total }}}{\partial z}\right\rangle\right|_{z=0}, \\
J=-\left.\kappa_{S}\left\langle\frac{\partial S_{\text {total }}}{\partial z}\right\rangle\right|_{z=0},
\end{gathered}
$$

where the angular brackets correspond to a horizontal average and

$$
\begin{aligned}
& T_{\text {total }}=T_{0}-\Delta T \frac{z}{d}+T(x, z, t), \\
& S_{\text {total }}=S_{0}-\Delta S \frac{z}{d}+S(x, z, t) .
\end{aligned}
$$

Substituting (32)-(34) into (48) and using the resultant equation (48) yield

$$
\begin{gathered}
H=\frac{\kappa_{T} \Delta T}{d}\left(1-2 \pi B_{2}\right), \\
J=\frac{\kappa_{S} \Delta S}{d}\left(1-2 \pi C_{2}\right) .
\end{gathered}
$$

The Nusselt and Sherwood numbers are defined by

$$
\begin{aligned}
& \mathrm{Nu}=\frac{H d}{\kappa_{T} \Delta T}=1-2 \pi B_{2}, \\
& \mathrm{Sh}=\frac{J d}{\kappa_{S} \Delta S}=1-2 \pi C_{2} .
\end{aligned}
$$

Substituting $B_{2}$ and $C_{2}$ of (41) into (51) gives

$$
\begin{gathered}
\mathrm{Nu}=1+\frac{4 \pi^{2} a^{2} F(z) A_{1}^{2}}{2\left(4 \pi^{2}-R_{i}\right)\left(R_{i}-\delta^{2}\right)-\pi^{2} a^{2} A_{1}^{2}}, \\
\mathrm{Sh}=1+\frac{2 a^{2} \mathrm{Le}^{2} A_{1}^{2}}{8 \delta^{2}+a^{2} \mathrm{Le}^{2} A_{1}^{2}} .
\end{gathered}
$$

\section{Results and Discussion}

5.1. Linear Stability Analysis. The linear stability analysis of double diffusive convection in a binary Maxwell fluid saturated porous layer with internal heat source has been studied analytically. In this section, we discuss the effects of the parameters in the governing equations on the onset of the double diffusive convection numerically and graphically. Figure 1 shows the neutral curves for stationary and oscillatory mode for fixed values of $\mathrm{Va}=12, \mathrm{Ra}_{S}=$ 500, $\lambda=0.01, R_{i}=3$, Le $=7$, and $\eta=0.8$ with variation in one of the parameters. From Figure 1, it can be found that the increase in the value of normalized porosity parameter $\eta$, stress relaxation time $\lambda$, and Lewis number Le decreases the oscillatory critical Rayleigh number. This indicates that those parameters advance the onset of double diffusive convection. Figure 1(d) depicts the stationary Rayleigh number increases with an increasing solutal Rayleigh number $\mathrm{Ra}_{S}$, which indicates that the effect of the solutal Rayleigh number is to enhance the stability of the system. On the other hand, the oscillatory Rayleigh number decreases with the increasing solutal Rayleigh number, which means that the solutal Rayleigh number has a stabilizing effect on the oscillatory onset. In Figure 1(f), we observe that the stationary Rayleigh number decreases with the increase in internal Rayleigh number $R_{i}$, which indicates that the internal Rayleigh number destabilizes the system. Additionally, the increasing internal Rayleigh number decreases the minimum of oscillatory Rayleigh number, which means that the internal Rayleigh number has a destabilizing effect on the oscillatory onset. Figure 1(e) shows the effect of Vadasz number on the critical Rayleigh number of stationary mode for fixed values of other parameters. When $a$ is small, the increasing $\mathrm{Va}$ decreases the critical Rayleigh number, indicating that the 


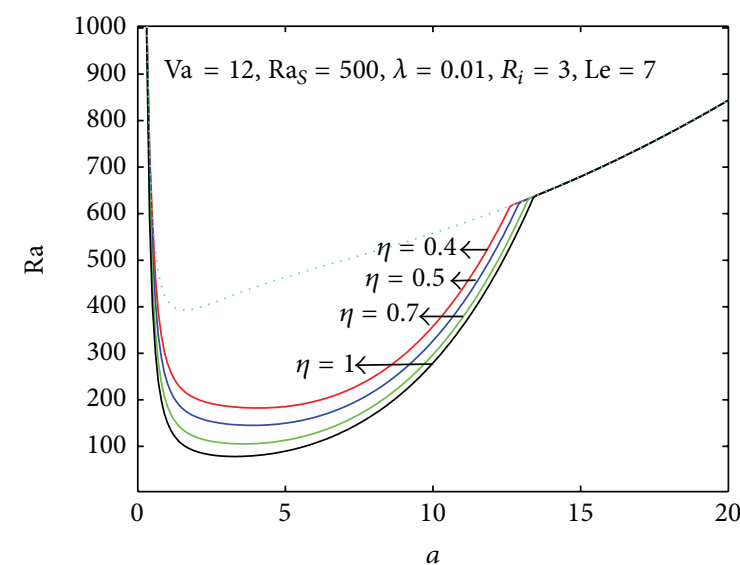

_ Oscillatory Stationary

(a)

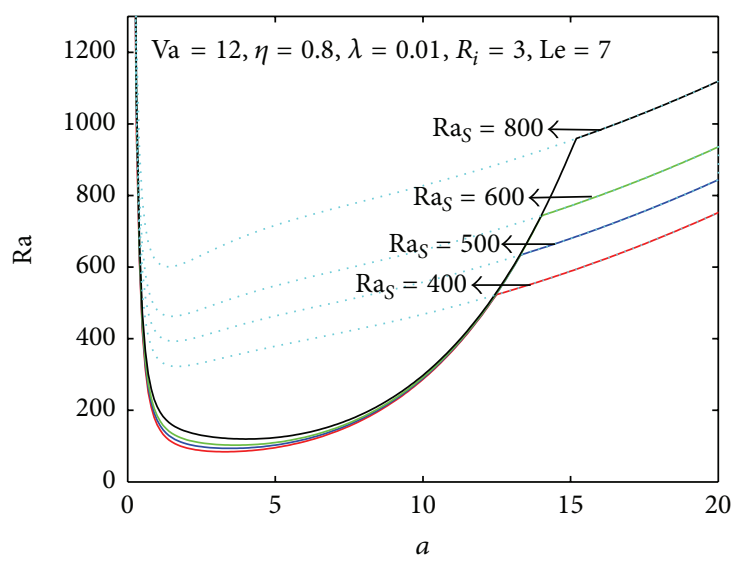

_- Oscillatory

Stationary

(c)

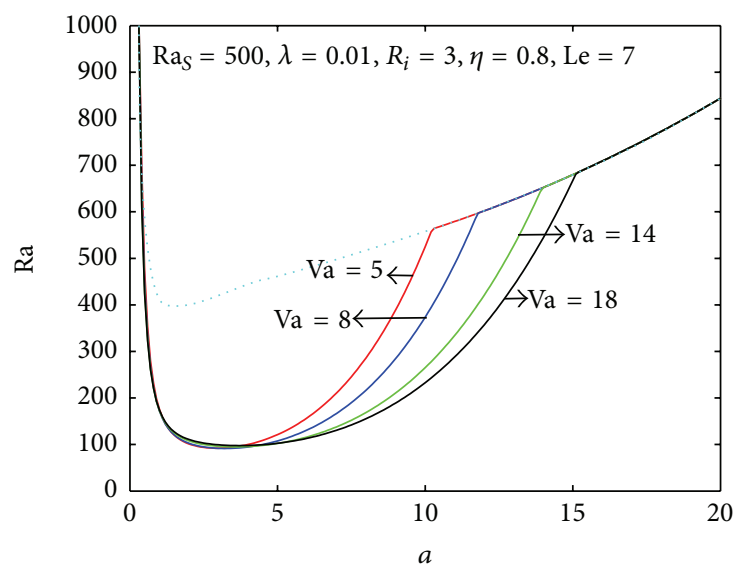

Oscillatory

Stationary

(e)

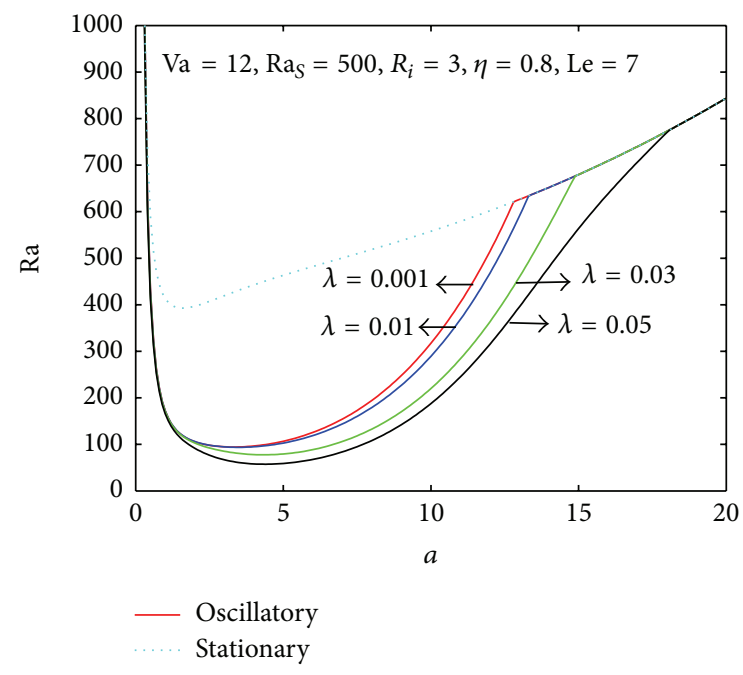

(b)

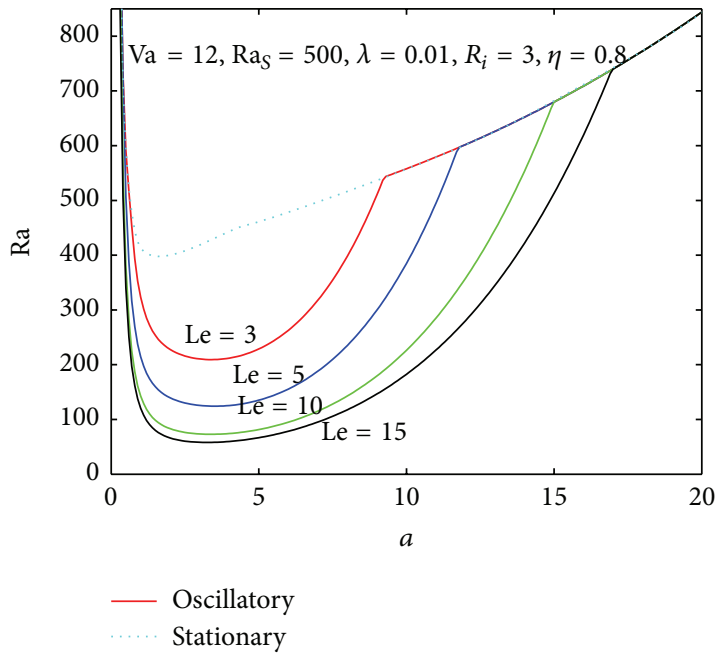

(d)

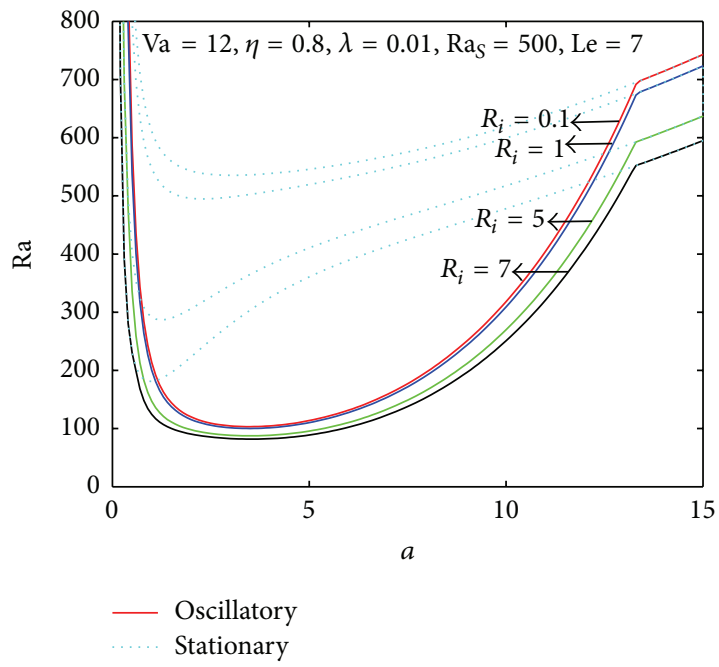

(f)

FIGURE 1: Variation of $\mathrm{Ra}_{T}$ with respect to wave number $a$ for neutral stability curves for different values of parameters. 

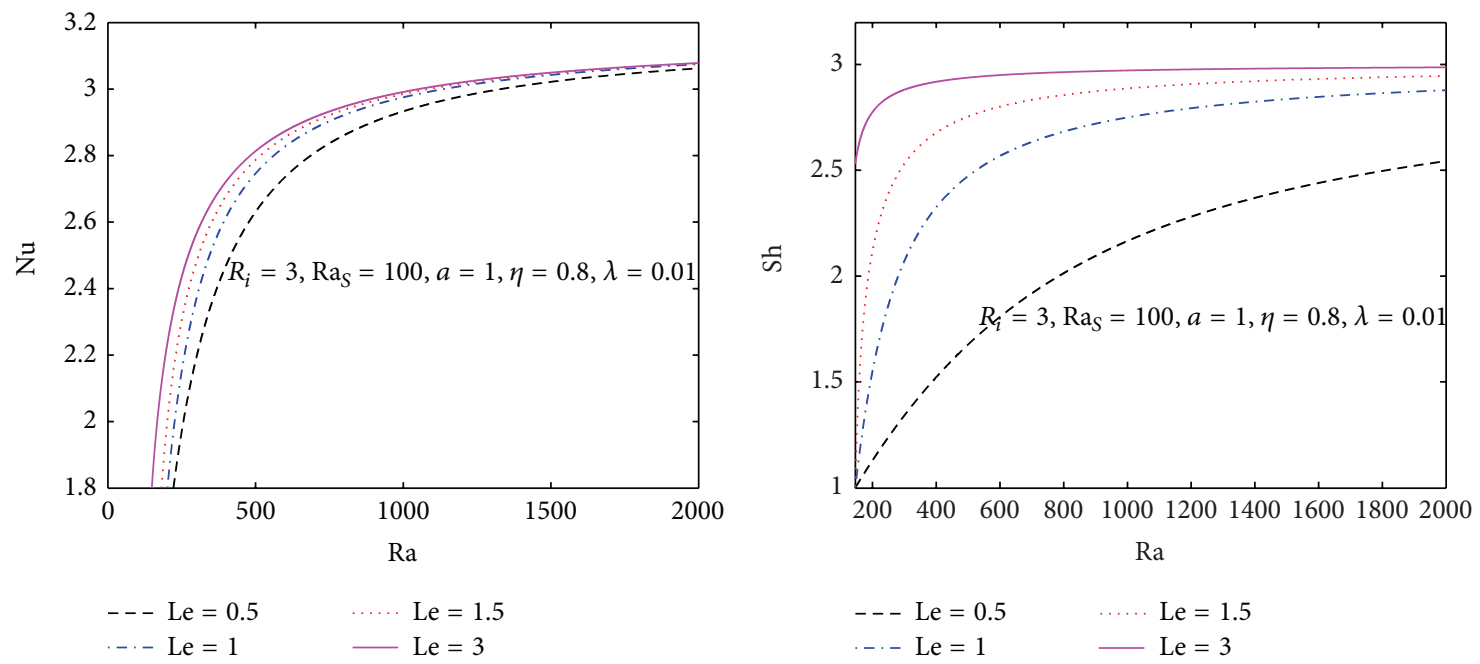

(a)
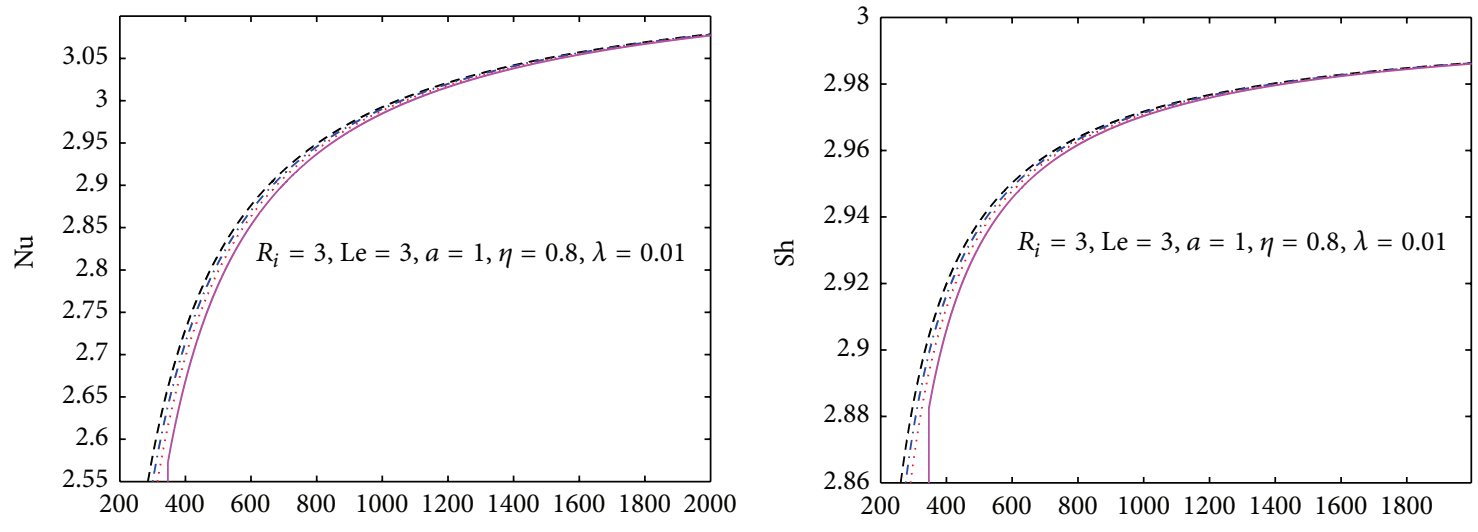

$\mathrm{Ra}$

$\mathrm{Ra}$

$$
\begin{aligned}
---\mathrm{Ra}_{S}=50 & \ldots \mathrm{Ra}_{S}=250 \\
--\mathrm{Ra}_{S} & =150 \quad-\mathrm{Ra}_{S}=400
\end{aligned}
$$

(c)

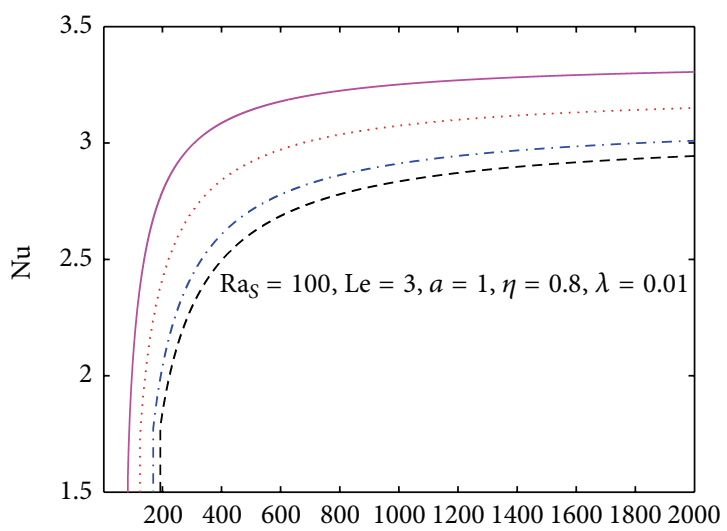

$\mathrm{Ra}$

$$
\begin{array}{ll}
--R_{i}=1 & \ldots . \cdot R_{i}=4 \\
--R_{i}=2 & -R_{i}=6
\end{array}
$$

(e)

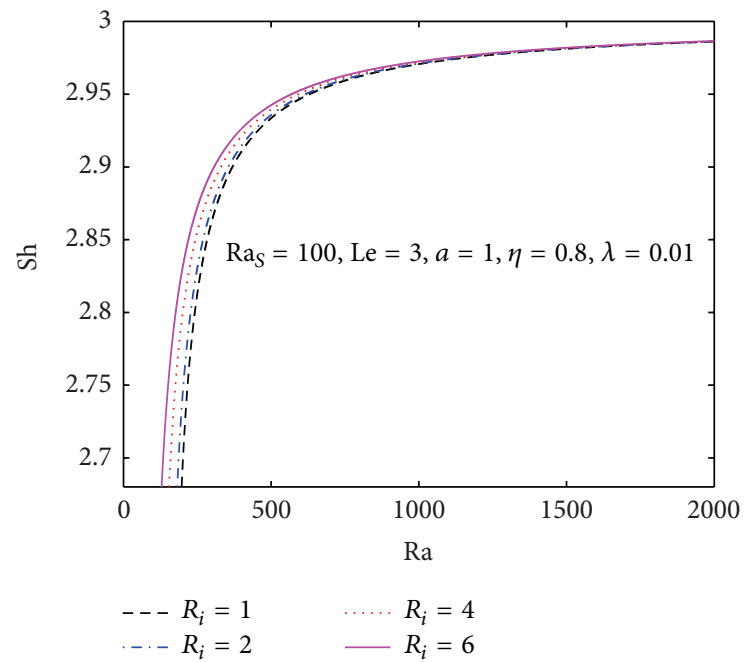

(f)

Figure 2: Variation in Nusselt number $\mathrm{Nu}$ and Sherwood number Sh with respect to Rayleigh number $\mathrm{Ra}_{T}$ for steady nonlinear stability at different values of parameters. 


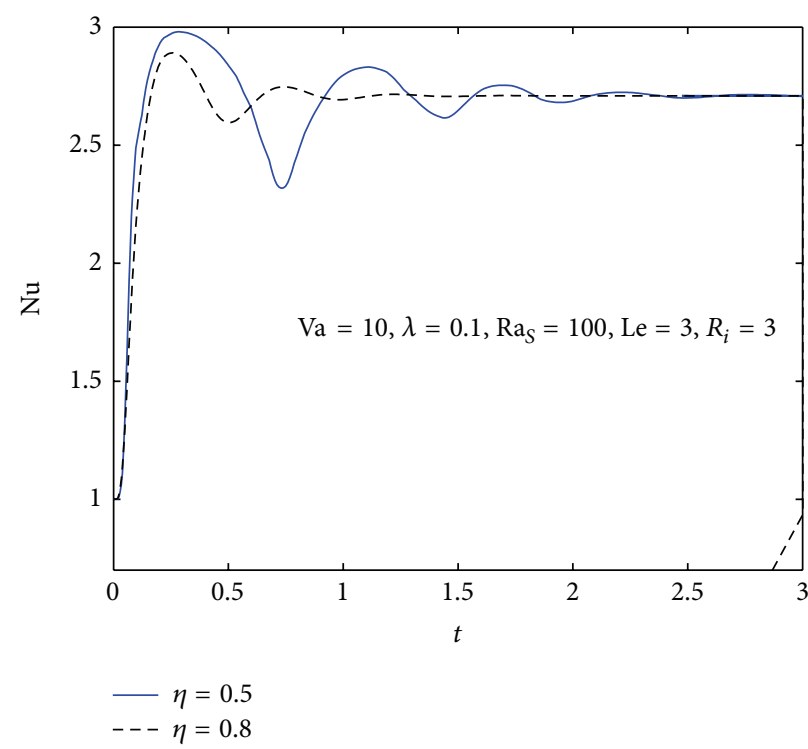

(a)

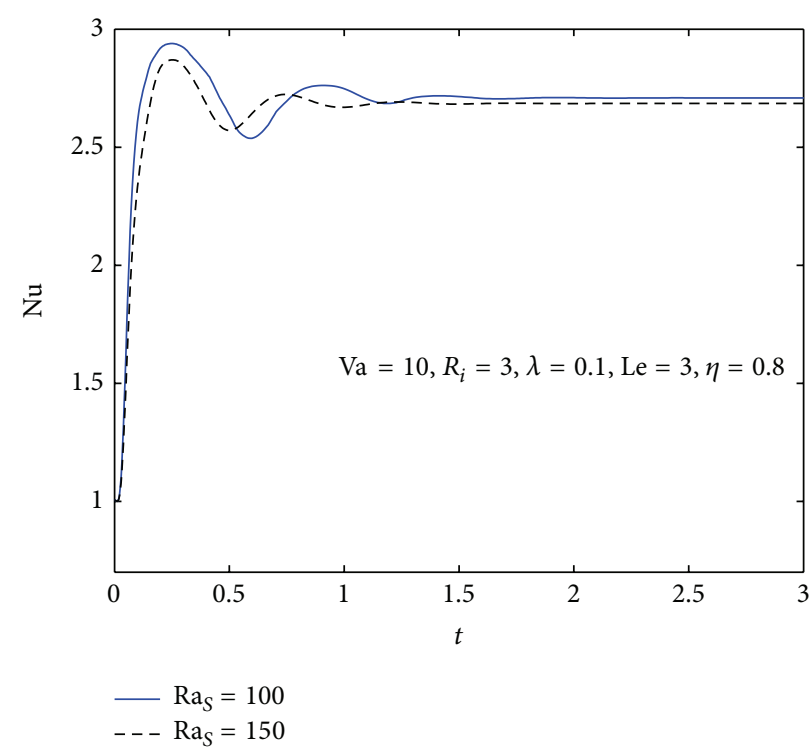

(c)

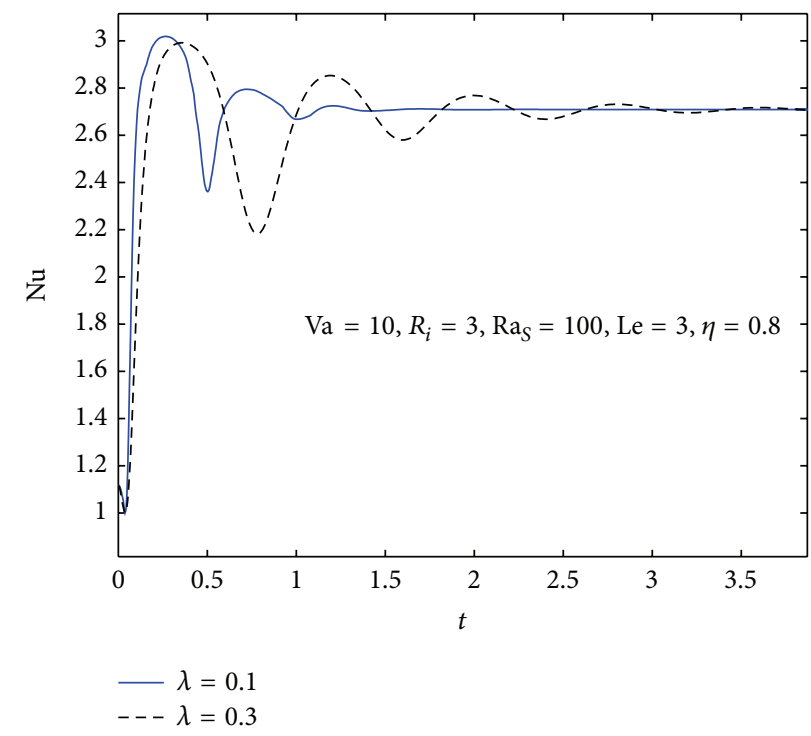

(b)

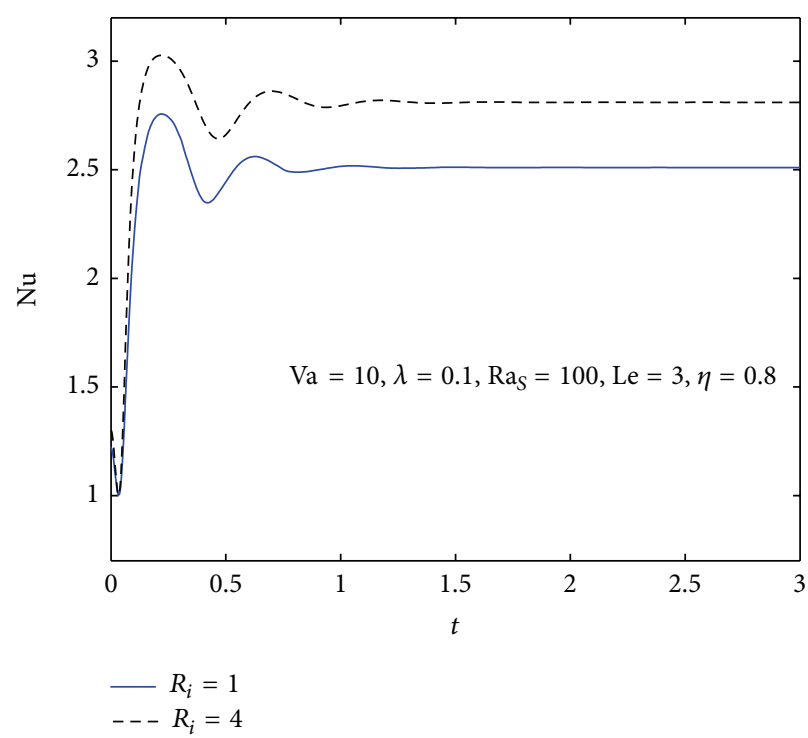

(d)

FIGURE 3: Variation in Nusselt number Nu with respect to time $t$ for unsteady nonlinear stability at different values of parameters.

effect of the increasing Va is to stabilize the system. However, for large value of the wavenumber, the facts are just the opposite.

5.2. Nonlinear Analysis. The weak nonlinear analysis provides the quantification of heat and mass transport. The effects of various parameters on the rate of heat and mass transfer are shown in Figure 2. The values of parameters are fixed at $R_{i}=3, \mathrm{Ra}_{S}=100, \eta=0.8, \lambda=0.01$, and $\mathrm{Le}=3$ with variation in one of the parameters. Figures $2(a)$ and $2(b)$ show that an increase in the value of Lewis number Le increases the value of $\mathrm{Nu}$ and $\mathrm{Sh}$. Thus, the effect of Lewis number is to increase the rate of heat and mass transfer.
Figures 2(c) and 2(d) present that both the rate of heat and mass transfer decrease with the increasing solutal Rayleigh number. Figures 2(e) and 2(f) show that an increase in the value of the internal Rayleigh number $R_{i}$ increases both the rate of heat and mass transfer.

Using the Runge-Kutta method with suitable initial conditions, we solve the autonomous system numerically given by (37). For fixed parameters $R_{i}=3, \mathrm{Ra}_{S}=100, \eta=$ $0.8, \lambda=0.1$, and Le $=3$ with variation in one of the parameters, the results of Nusselt number $\mathrm{Nu}$ and Sherwood number Sh with respect to time $t$ are graphically shown in Figures 3 and 4, respectively. Although the maximum amplification of $\mathrm{Nu}$ and $\mathrm{Sh}$ occurs at the beginning time, it generates spatial oscillations of increasing frequency. Eventually 


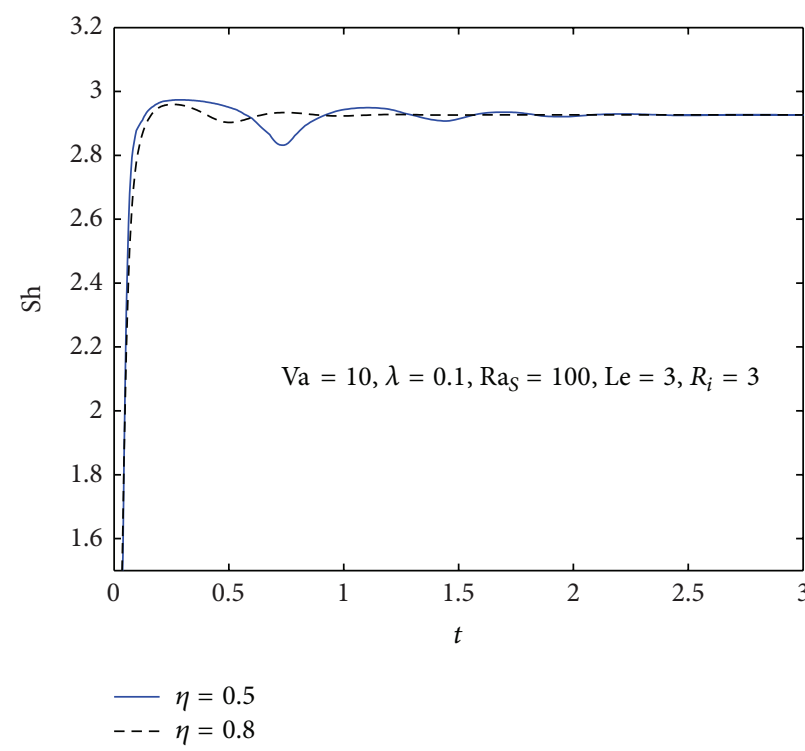

(a)

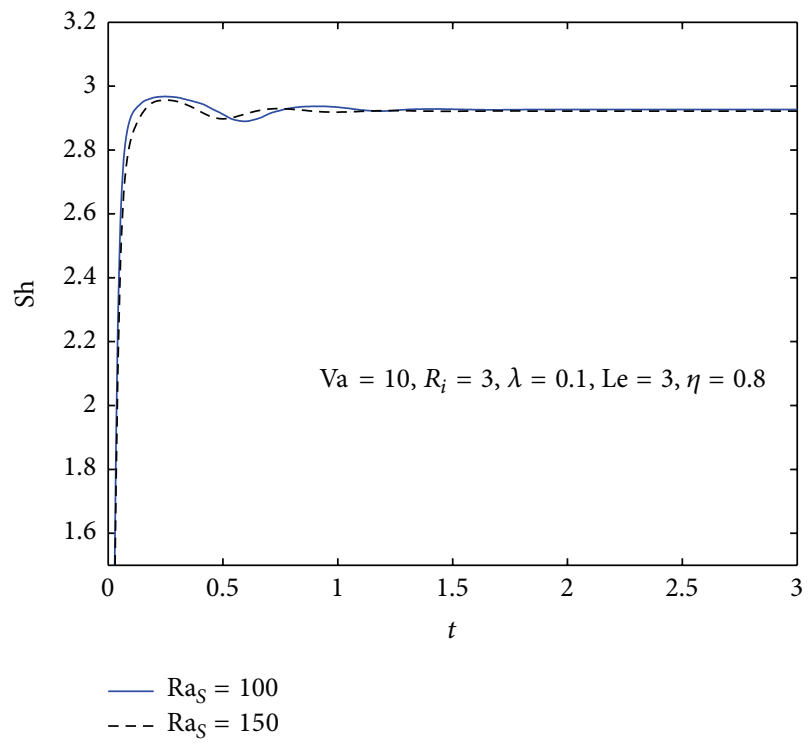

(c)

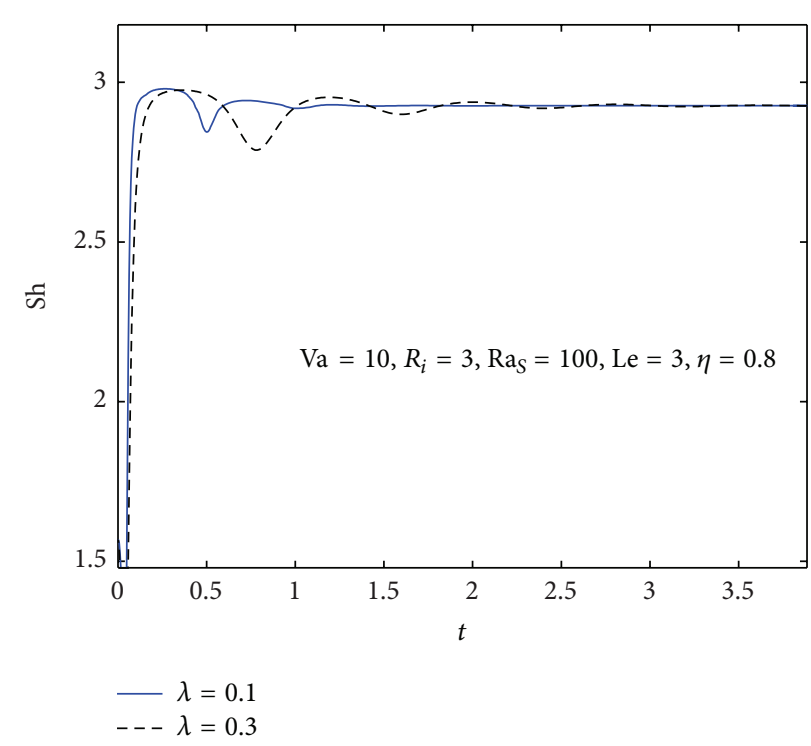

(b)

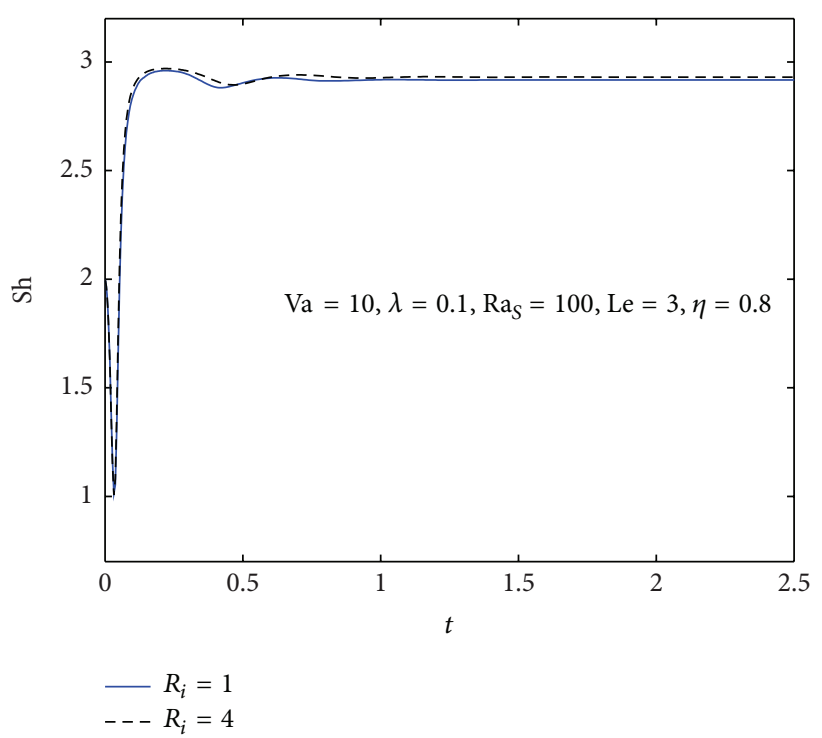

(d)

FIGURE 4: Variation in Nusselt number Sh with respect to time $t$ for unsteady nonlinear stability at different values of parameters.

the oscillations reach a steady state when time is large enough. Figures 3 and 4 show that the parameters have insignificant effect on heat transfer; however, the effects of the parameters on mass transfer are smaller. Notice by inspection of Figures 3 and 4 that $\mathrm{Nu}$ and $\mathrm{Sh}$ are considerably reduced as the value of relaxation time $\lambda$ increases, and so are $\eta$ and $R_{S}$. However, the internal Rayleigh number $\mathrm{Ra}_{i}$ increases the rate of heat and mass transfer.

\section{Conclusion}

Linear and nonlinear analysis of double diffusive convection in a Maxwell fluid saturated porous layer with internal heat source, which is heated and salted from below, is investigated analytically and numerically. The linear analysis is analyzed using the normal mode technique. On the other hand, the nonlinear analysis of the system is established through a truncated form of the Fourier series. The effects of physical parameters in governing equations, such as relaxation time, Lewis number, normalized porosity parameter, Vadasz number, solutal Rayleigh number, and internal Rayleigh number, on stationary, oscillatory convection, and heat and mass transfer are shown graphically and the following conclusions are drawn: Vadasz number Va, relaxation parameter $\lambda$, and Lewis number Le advance the onset of oscillatory convection. The internal Rayleigh number $R_{i}$ has a destabilizing effect for the governing system, both stationary and oscillatory mode, and the solutal Rayleigh number $\mathrm{Ra}_{S}$ stabilizes the system. The rate heat and mass transfer increase with 
the increasing internal Rayleigh number, but $\mathrm{Nu}$ and $\mathrm{Sh}$ are reduced as the solutal Rayleigh number, stress relaxation time, and normalized porosity parameter increase.

\section{Conflict of Interests}

The authors declare that there is no conflict of interests regarding the publication of this paper.

\section{Acknowledgments}

This work is supported by the National Natural Science Foundation of China (nos. 11002083, 51279093, and $41172268)$ and the National Basic Research Program of China (2013CB036000).

\section{References}

[1] J. Azaiez and M. Sajjadi, "Stability of double-diffusive doubleconvective miscible displacements in porous media," Physical Review E, vol. 85, no. 2, Article ID 026306, 2012.

[2] N. Rudraiah and P. G. Siddheshwar, "A weak nonlinear stability analysis of double diffusive convection with cross-diffusion in a fluid-saturated porous medium," Heat and Mass Transfer, vol. 33, no. 4, pp. 287-293, 1998.

[3] I. S. Shivakumara, J. Lee, S. Suresh Kumar, and N. Devaraju, "Linear and nonlinear stability of double diffusive convection in a couple stress fluid-saturated porous layer," Archive of Applied Mechanics, vol. 81, no. 11, pp. 1697-1715, 2011.

[4] O. V. Trevisan and A. Bejan, "Combined heat and mass transfer by natural convection in a porous medium," Advances in Heat Transfer, vol. 20, pp. 315-352, 1990.

[5] C. Zhao, B. E. Hobbs, H. B. Mühlhaus, A. Ord, and G. Lin, "Numerical modelling of double diffusion driven reactive flow transport in deformable fluid-saturated porous media with particular consideration of temperature-dependent chemical reaction rates," Engineering Computations, vol. 17, no. 4, pp. 367$385,2000$.

[6] C. Zhao, B. E. Hobbs, A. Ord, S. Peng, H. B. Mühlhaus, and L. Liu, "Double diffusion-driven convective instability of threedimensional fluid-saturated geological fault zones heated from below," Mathematical Geology, vol. 37, no. 4, pp. 373-391, 2005.

[7] C. Zhao, B. E. Hobbs, A. Ord, M. Kühn, H. B. Mühlhaus, and S. Peng, "Numerical simulation of double-diffusion driven convective flow and rock alteration in three-dimensional fluidsaturated geological fault zones," Computer Methods in Applied Mechanics and Engineering, vol. 195, no. 19-22, pp. 2816-2840, 2006.

[8] C. Zhao, B. E. Hobbs, and A. Ord, Convective and Advective Heat Transfer in Geological Systems, Springer, Berlin, Germany, 2008.

[9] C. Zhao, B. E. Hobbs, and A. Ord, Fundamentals of Computational Geoscience: Numerical Methods and Algorithms, Springer, Berlin, Germany, 2009.

[10] D. A. Nield and A. Bejan, Convection in Porous Media, Springer, New York, NY, USA, 3rd edition, 2006.

[11] D. B. Ingham and I. Pop, Transport Phenomena in Porous Media, vol. III, Elsevier, Oxford, UK, 2005.

[12] K. Vafai, Handbook of Porous Media, Taylor and Francis, London, UK, CRC Press, Boca Raton, Fla, USA, 2005.
[13] C. W. Horton and F. T. Rogers Jr., "Convection currents in a porous medium," Journal of Applied Physics, vol. 16, pp. 367-370, 1945.

[14] E. R. Lapwood, "Convection of a fluid in a porous medium," Proceedings of the Cambridge Philosophical Society, vol. 44, pp. 508-521, 1948 .

[15] C. Zhao, H. B. Mühlhaus, and B. E. Hobbs, "Finite element analysis of steady-state natural convection problems in fluidsaturated porous media heated from below," International Journal for Numerical and Analytical Methods in Geomechanics, vol. 21, no. 12, pp. 863-881, 1997.

[16] C. Zhao, B. E. Hobbs, and H. B. Mühlhaus, "Finite element modelling of temperature gradient driven rock alteration and mineralization in porous rock masses," Computer Methods in Applied Mechanics and Engineering, vol. 165, no. 1-4, pp. 175187, 1998.

[17] C. Zhao, H. B. Mühlhaus, and B. E. Hobbs, "Effects of geological inhomogeneity on high rayleigh number steady state heat and mass transfer in fluid-saturated porous media heated from below," Numerical Heat Transfer A, vol. 33, no. 4, pp. 415-431, 1998.

[18] C. Zhao, B. E. Hobbs, and H. B. Mühlhaus, "Theoretical and numerical analyses of convective instability in porous media with upward throughflow," International Journal for Numerical and Analytical Methods in Geomechanics, vol. 23, no. 7, pp. 629646, 1999.

[19] C. Zhao, B. E. Hobbs, and H. B. Mühlhaus, "Effects of medium thermoelasticity on high Rayleigh number steady-state heat transfer and mineralization in deformable fluid-saturated porous media heated from below," Computer Methods in Applied Mechanics and Engineering, vol. 173, no. 1-2, pp. 41-54, 1999.

[20] C. Zhao, B. E. Hobbs, H. B. Mühlhaus, A. Ord, and G. Lin, "Finite element modelling of three-dimensional convection problems in pore-fluid saturated porous media heated from below," Communications in Numerical Methods in Engineering, vol. 17, pp. 101-114, 2001.

[21] C. Zhao, B. E. Hobbs, H. B. Mühlhaus, A. Ord, and G. Lin, "Convective instability of 3-D fluid-saturated geological fault zones heated from below, Geophysical Journal International, vol. 155, no. 1, pp. 213-220, 2003.

[22] C. Zhao, B. E. Hobbs, A. Ord, H. B. Mühlhaus, and G. Lin, "Effect of material anisotropy on the onset of convective flow in three-dimensional fluid-saturated faults," Mathematical Geology, vol. 35, no. 2, pp. 141-154, 2003.

[23] C. Zhao, B. E. Hobbs, A. Ord, S. Peng, H. B. Mühlhaus, and L. Liu, "Theoretical investigation of convective instability in inclined and fluid-saturated three-dimensional fault zones," Tectonophysics, vol. 387, no. 1-4, pp. 47-64, 2004.

[24] C. Parthiban and P. R. Patil, "Thermal instability in an anisotropic porous medium with internal heat source and inclined temperature gradient," International Communications in Heat and Mass Transfer, vol. 24, no. 7, pp. 1049-1058, 1997.

[25] B. Straughan and J. Tracey, "Multi-component convectiondiffusion with internal heating or cooling," Acta Mechanica, vol. 133, no. 1-4, pp. 219-238, 1999.

[26] E. Magyari, I. Pop, and A. Postelnicu, "Effect of the source term on steady free convection boundary layer flows over a vertical plate in a porous medium. I," Transport in Porous Media, vol. 67, pp. 49-67, 2007.

[27] E. Magyari, I. Pop, and A. Postelnicu, "Effect of the source term on steady free convection boundary layer flows over a vertical 
plate in a porous medium. II," Transport in Porous Media, vol. 67, no. 2, pp. 189-201, 2007.

[28] A. A. Hill, "Double-diffusive convection in a porous medium with a concentration based internal heat source," Proceedings of The Royal Society of London A, vol. 461, no. 2054, pp. 561-574, 2005.

[29] B. S. Bhadauria, A. Kumar, J. Kumar, N. C. Sacheti, and P. Chandran, "Natural convection in a rotating anisotropic porous layer with internal heat generation," Transport in Porous Media, vol. 90, no. 2, pp. 687-705, 2011.

[30] B. S. Bhadauria, "Double-diffusive convection in a saturated anisotropic porous layer with internal heat source," Transport in Porous Media, vol. 92, no. 2, pp. 299-320, 2012.

[31] S. Wang and W. Tan, "Stability analysis of double-diffusive convection of Maxwell fluid in a porous medium heated from below," Physics Letters A, vol. 372, no. 17, pp. 3046-3050, 2008.

[32] S. N. Gaikwad, M. S. Malashetty, and K. Rama Prasad, "An analytical study of linear and nonlinear double diffusive convection in a fluid saturated anisotropic porous layer with Soret effect," Applied Mathematical Modelling, vol. 33, no. 9, pp. 3617-3635, 2009.

[33] S. N. Gaikwad, M. S. Malashetty, and K. Rama Prasad, "Linear and non-linear double diffusive convection in a fluid-saturated anisotropic porous layer with cross-diffusion effects," Transport in Porous Media, vol. 80, no. 3, pp. 537-560, 2009.

[34] M. C. Kim, S. B. Lee, S. Kim, and B. J. Chung, "Thermal instability of viscoelastic fluids in porous media," International Journal of Heat and Mass Transfer, vol. 46, no. 26, pp. 50655072, 2003.

[35] M. S. Malashetty and B. S. Biradar, "The onset of double diffusive convection in a binary Maxwell fluid saturated porous layer with cross-diffusion effects," Physics of Fluids, vol. 23, no. 6, Article ID 064109, 2011.

[36] M. S. Malashetty, W. Tan, and M. Swamy, "The onset of double diffusive convection in a binary viscoelastic fluid saturated anisotropic porous layer," Physics of Fluids, vol. 21, no. 8, Article ID 084101, 2009. 


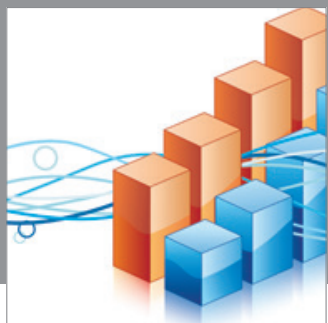

Advances in

Operations Research

mansans

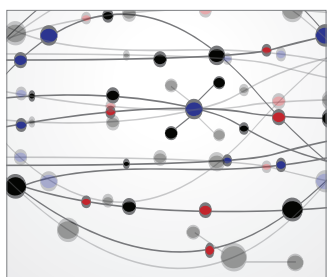

The Scientific World Journal
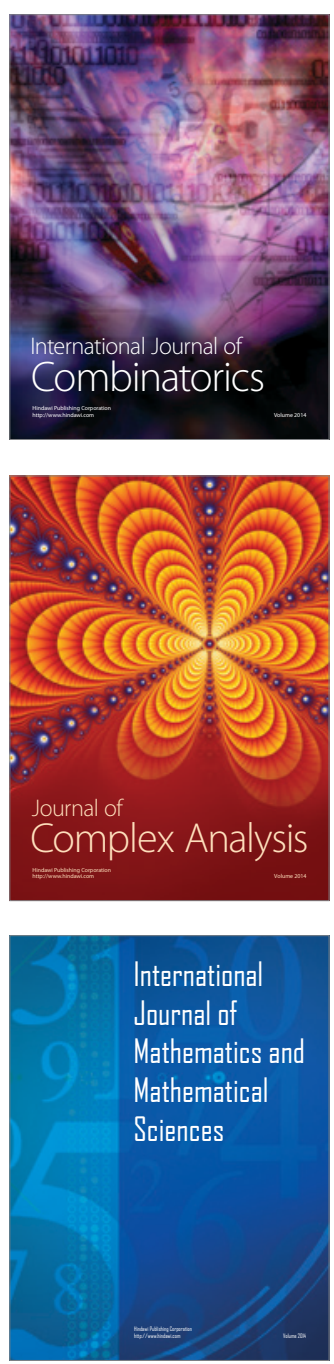
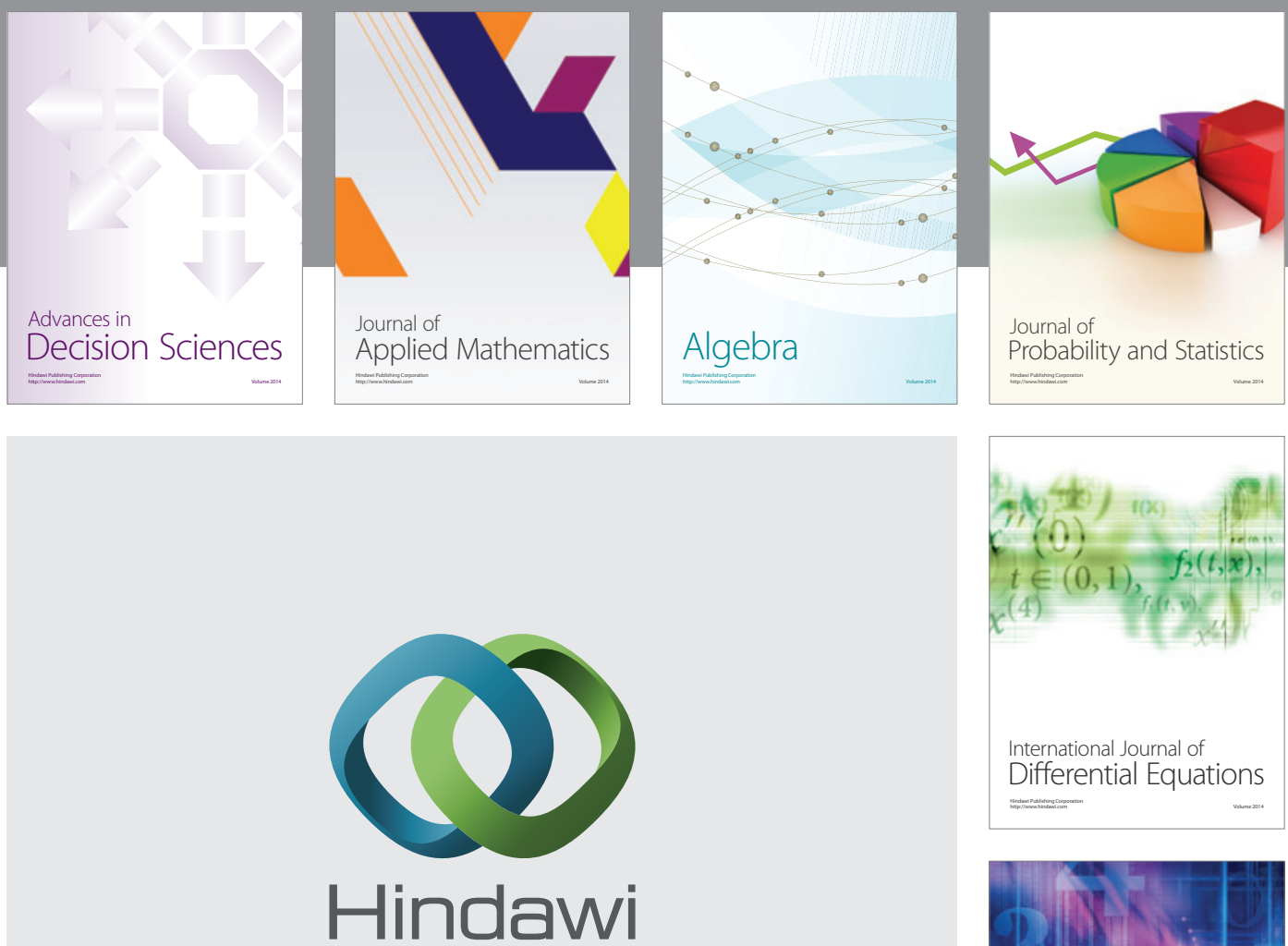

Submit your manuscripts at http://www.hindawi.com
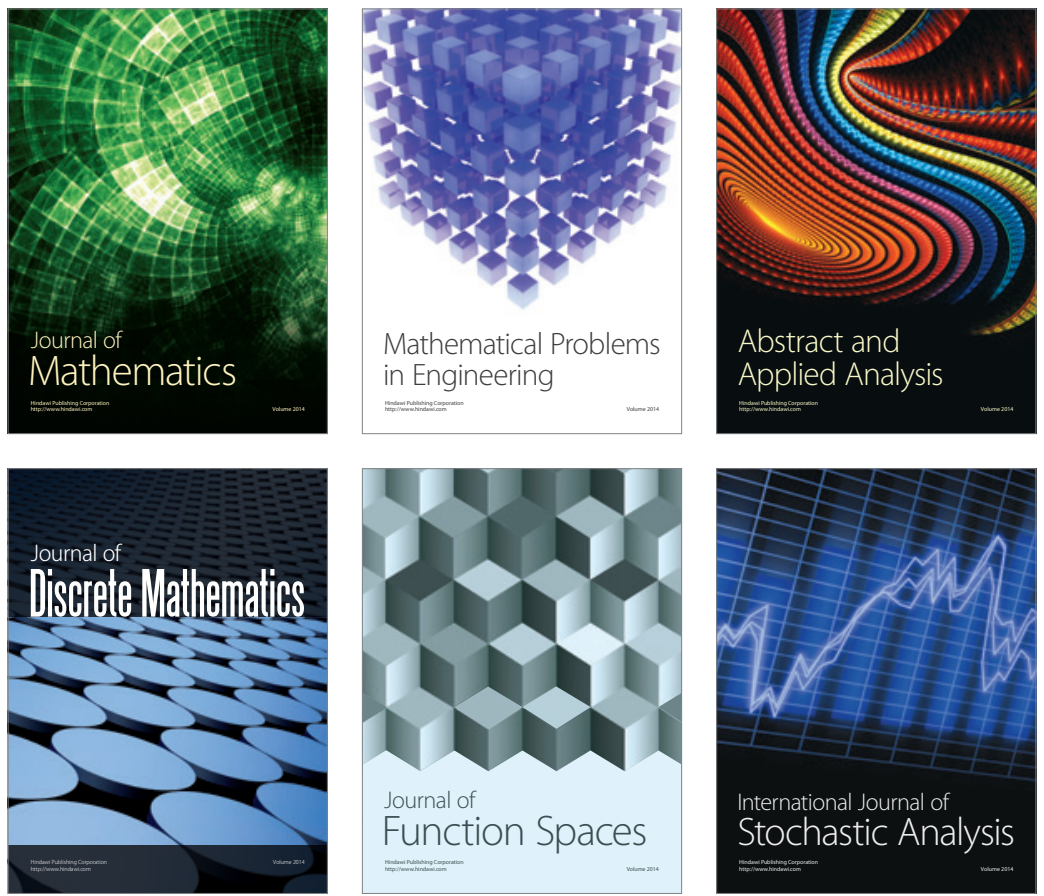

Journal of

Function Spaces

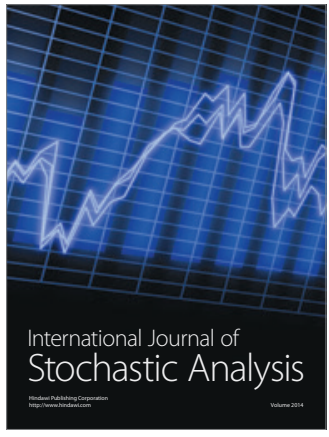

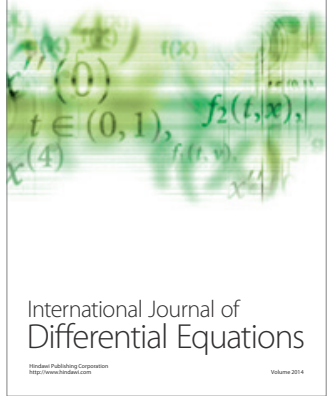
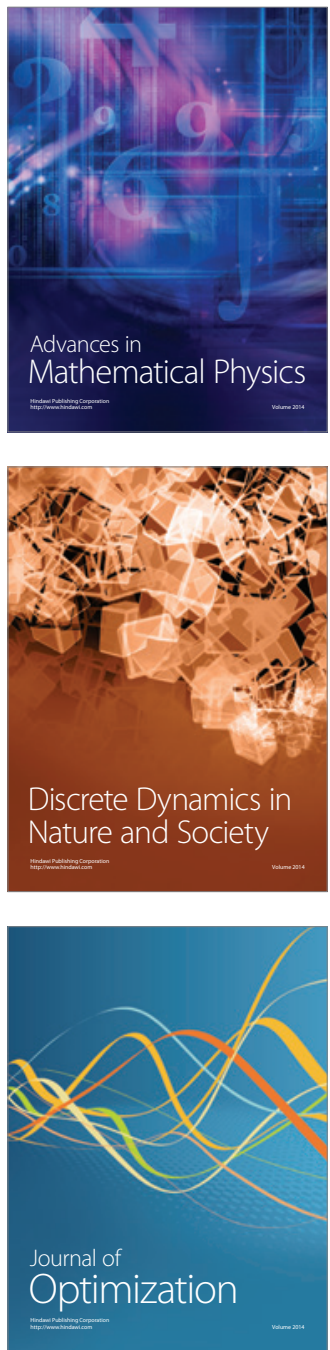